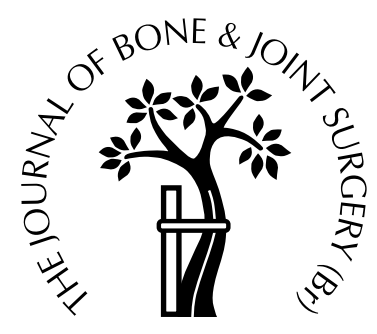

\title{
Combined cuboid/cuneiform osteotomy for correction of residual adductus deformity in idiopathic and secondary club feet
}

\author{
Dirk Schaefer, Fritz Hefti \\ From the University of Basel, Switzerland
}

$\mathbf{W}$ e used a combined cuboid/cuneiform osteotomy to treat residual adductus deformity in idiopathic and secondary club feet. The mean follow-up for 27 feet (22 idiopathic, four arthrogrypotic and one related to amniotic band syndrome) was 5.0 years $(2.0$ to 9.8$)$. All healed uneventfully except for one early wound infection. No further surgery was required in the 22 idiopathic club feet but four of five with secondary deformity needed further surgery. At follow-up all patients with idiopathic and two with secondary club feet were free from pain and satisfied with the result. In the idiopathic feet, adductus of the forefoot, as measured by the calcaneal second metatarsal angle, improved on average from $20.7 \pm 2.0^{\circ}$ to $8.9 \pm 1.8^{\circ}(p<0.05)$. In four feet, with a follow-up of more than six years, there was complete recurrence of the deformity. In the secondary club feet, there was no improvement of the adductus. We conclude that in most, but not all, idiopathic club feet a cuboid/cuneiform osteotomy can provide satisfactory correction of adductus deformity. Those with secondary deformity require other procedures.

J Bone Joint Surg [Br] 2000;82-B:881-4. Received 14 December 1999; Accepted 10 February 2000

Recurrent adductus of the forefoot is commonly seen in treated idiopathic club feet, usually in children over three years of age. ${ }^{1}$ Tarraf and Carroll ${ }^{2}$ in an analysis of residual deformity in a series of 159 club feet found adduction in $81.1 \%$ at the first revision and in $47.5 \%$ at the second revision. Undercorrection at the time of the initial surgery and medial displacement of the anterior part of the calca-

D. Schaefer, MD

F. Hefti, MD, Professor

Children's Orthopaedic Department, University of Basel, Roemergasse 8, CH-4005 Basel, Switzerland.

Correspondence should be sent to Dr D. Schaefer.

(C)2000 British Editorial Society of Bone and Joint Surgery 0301-620X/00/610845\$2.00 neus and the navicular around the talus were considered to be aetiological factors. Muscular imbalance between the abductors and adductors of the foot and abnormal attachments of the tendon of tibialis anterior have been found in these feet. ${ }^{3}$ Capsulotomies of the tarsometatarsal joints has been advocated after failed conservative treatment but an incidence of degenerative joint disease of $68 \%$ has been reported. $^{4}$ Soft-tissue revision surgery is more difficult because of scarring from previous operations and does not take into account deformation of the midtarsal bones which occurs with time. ${ }^{3}$

The combination of a shortening osteotomy of the cuboid and elongation of the cuneiform, as first described by McHale and Lenhart, ${ }^{5}$ has the potential to correct the deformity of the tarsal bones with minimal additional scarring. It is not known whether this operation can maintain correction of forefoot adduction during growth. We report our experience with this double osteotomy with a mean follow-up of five years.

\section{Patients and Methods}

Out of 27 club feet, 22 (16 patients) were classified as idiopathic, four (three patients) as associated with arthrogryposis and was due to an amniotic band syndrome. All had previously undergone a full posteromedial/posterolateral release of the ankle and subtalar joints, with lengthening of tendo Achillis. In idiopathic club feet, seven osteotomies of the tibia, three medial plantar releases, one lengthening of the posterior tibial tendon and one plantar fasciotomy had been carried out, in addition to treatment by casts and braces before the planned operation. In the secondary club feet one open reduction of the talus and two medial releases of soft-tissue had been undertaken.

Cuboid/cuneiform osteotomy was performed at a mean age of 9.5 years (4.1 to 20.0) in the period from October 1985 to March 1994. Under general anaesthesia, the cuboid and medial cuneiform were identified using an image intensifier. Through a lateral longitudinal incision over the cuboid, a bony wedge based laterally was removed from the cuboid and inserted through a medial incision into an osteotomy of the medial cuneiform with its base medially. ${ }^{5}$ The wedge had a width of approximately one-third that of the cuboid on its lateral side. In idiopathic club feet, a 
Table I. The mean $( \pm \mathrm{SD})$ preoperative and follow-up measurements at five years for the 27 feet which underwent cuboid/cuneiform osteotomy

\begin{tabular}{lcccc}
\hline & Preoperative & Follow-up & Difference & p value \\
\hline Range of ankle movement (degrees) & $38.9 \pm 3.2$ & $48.2 \pm 3.0$ & 9.3 & $\leq 0.05$ \\
Hindfoot forefoot angle (degrees) & $21.9 \pm 1.2$ & $10.0 \pm 2.4$ & -11.9 & $\leq 0.01$ \\
Lateral metatarsal height (cm) & $4.5 \pm 0.2$ & $5.0 \pm 0.2$ & 0.5 & $\leq 0.01$ \\
Lateral talar dome height (mm) & $4.0 \pm 0.8$ & $6.0 \pm 1.0$ & 2.0 & $\leq 0.01$ \\
Lateral talocalcaneal angle (degrees) & $24.8 \pm 1.4$ & $19.1 \pm 1.6$ & -5.7 & $\leq 0.01$ \\
Lateral talar first metatarsal angle (degrees) & $15.8 \pm 2.5$ & $18.6 \pm 4.6$ & 2.8 & \\
Talocalcaneal angle (degrees) & $7.3 \pm 0.8$ & $5.8 \pm 0.9$ & -1.5 & \\
Talar first metatarsal angle (degrees) & $16.9 \pm 1.8$ & $7.0 \pm 1.9$ & 9.9 & $\leq 0.01$ \\
AP calcaneal second metatarsal angle (degrees) & $20.7 \pm 2.0$ & $8.9 \pm 1.8$ & 11.8 & $\leq 0.01$ \\
\hline
\end{tabular}

medial release was added in three feet and a plantar fasciotomy in one. In the secondary feet two medial softtissue releases were carried out. The osteotomies were transfixed with two Kirschner wires, one from the medial cuneiform into the navicular bone and the other from the cuboid into the calcaneus. Postoperatively, a long-leg nonweight-bearing cast was retained for six weeks.

At follow-up, all patients were specifically questioned about pain, mobility of the ankle, sporting activity and foot wear. The forefoot-hindfoot angle was measured on podograms. ${ }^{6}$ The talocalcaneal angle, the talar first metatarsal angle and the calcaneal second metatarsal angle were measured on weight-bearing anteroposterior (AP) radiographs. ${ }^{7}$ On lateral weight-bearing radiographs the talocalcaneal angle, the talar first metatarsal (Meary's) angle, the metatarsal height and the height of the talar dome were measured. ${ }^{8}$ Pre- and postoperative variables were compared using the Wilcoxon signed-rank test (SPSS, Chicago, Illinois). In 20 feet (16 idiopathic and four secondary) the AP calcaneal second metatarsal angle three months after operation was compared with that measured at follow-up in order to investigate whether correction had been lost after consolidation.

\section{Results}

The postoperative course was uneventful in all except one patient. He developed a wound infection after two weeks, which was successfully treated with antibiotics and softtissue surgery. All osteotomies had fully united after three months. No idiopathic club foot required further surgery. The secondary club feet subsequently needed two repeat cuboid/cuneiform osteotomies, one talectomy, one osteotomy of the calcaneus, one open reduction of the talus and one soft-tissue release ten months to seven years after the initial osteotomy because of recurrence of the deformity. At follow-up at a mean of five years (2.0 to 9.8) all patients with idiopathic club feet were free from pain and could walk at least $5 \mathrm{~km}$. All except one were able to wear normal shoes. Two feet needed insoles and one a custommade shoe. All except two patients were able to participate in competitive sports. The adductus deformity,

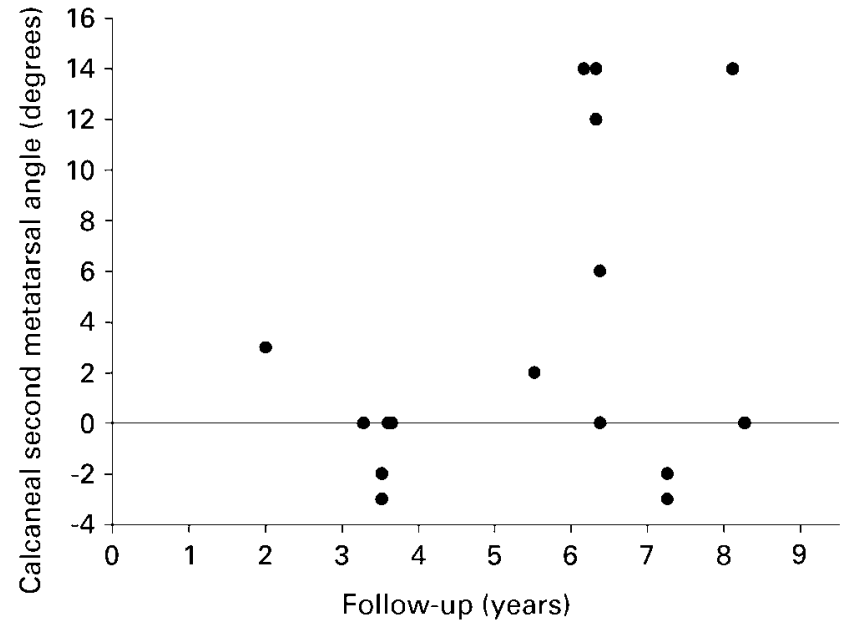

Fig. 1

Loss of correction in 16 idiopathic club feet. Dots represent the difference of the calcaneal second metatarsal angle measured three months postoperatively and at follow-up.

measured by the AP calcaneal second metatarsal angle, decreased significantly from a mean of $20.7 \pm 2.0^{\circ}$ to 4.6 $\pm 1.5^{\circ}$ after three months $(\mathrm{p}<0.05)$ and was $8.9 \pm 1.8^{\circ}$ at follow-up $(\mathrm{p}<0.05)$. In four of 16 idiopathic club feet there was nearly complete loss of correction (Fig. 1). All four had undergone previous tibial derotation osteotomy. Table I gives the preoperative and follow-up measurements. The mean range of ankle movement improved from $38.9^{\circ}$ to $48.2^{\circ}$. The hindfoot/forefoot angle, as measured on podography, decreased from $21.9^{\circ}$ to $10.0^{\circ}$. The lateral metatarsal height and that of the talar dome were increased at follow-up by 0.5 and $2 \mathrm{~mm}$, respectively. The lateral talocalcaneal angle was reduced before and after operation, indicating hindfoot malrotation. There were no signs of radiological joint degeneration at follow-up (Fig. 2).

In secondary club feet, three of the four patients ( 3 feet) suffered pain and two were unable to walk $100 \mathrm{~m}$. All required insoles, and two custom-made shoes. The AP calcaneal second metatarsal angle which measured $44.6 \pm$ $7.9^{\circ}$ preoperatively was $44.5 \pm 5.9^{\circ}$ after three months and $41.2 \pm 8.1^{\circ}$ at follow-up. 

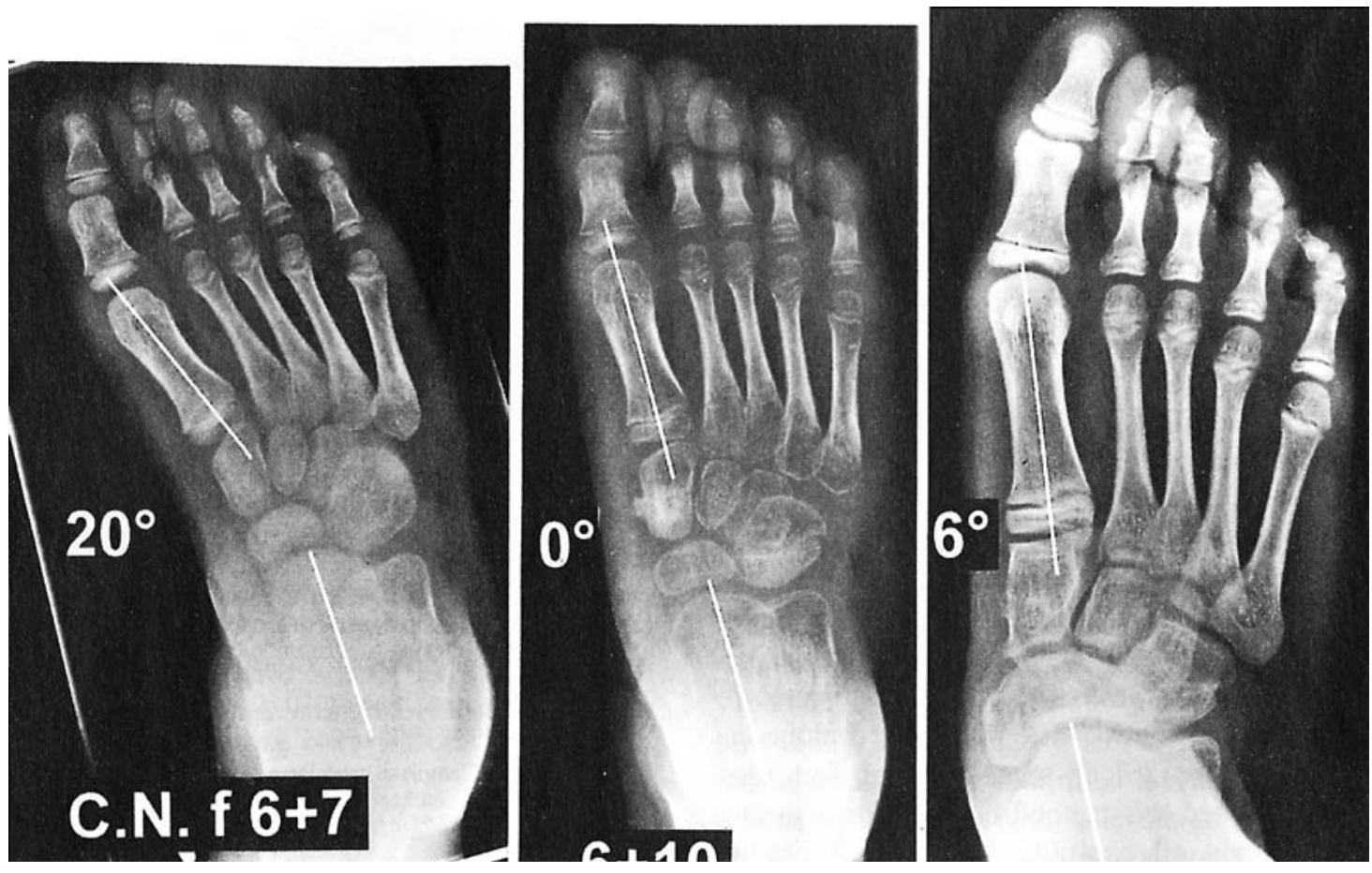

Fig. 2

Weight-bearing AP radiographs, before and at three months and 5.9 years after operation. The cuboid is shortened and the medial cuneiform lengthened thus correcting the adductus deformity. There is no evidence of degeneration in the midfoot joints.

\section{Discussion}

Revision procedures to correct adductus deformity in club feet increasingly require osteotomy as part of each subsequent procedure. Tarraf and Carroll ${ }^{2}$ at the first reoperation noted an incidence of soft-tissue operations of $46.5 \%$ compared with $4.4 \%$ of bony procedures, at the second revision $25 \%$ compared with $20 \%$, and at the third $27.3 \%$ compared with $54.5 \%$, the remainder being combinations of both. The increasing frequency of osteotomy was attributed to the presence of extensive scarring, making softtissue release more difficult. Scar tissue also promotes recurrence. Osteotomy also has the advantage of correcting deformities of the tarsal bones, which undergo deformation during growth in idiopathic club feet. ${ }^{9}$

The principle of combined cuboid/cuneiform osteotomy has been investigated in a cadaver study by McHale and Lenhart. ${ }^{5}$ Using metal wedges, the best position for the osteotomy, the influence of medial and lateral osteotomy, and the necessity for additional soft-tissue releases were examined. It was observed that the combination of both procedures gave the best correction compared with isolated osteotomies, that the correction was reproducible and that additional soft-tissue releases were not necessary. The experiment was, however, carried out on normal cadaver feet lacking the soft-tissue rigidity of a club foot. The procedure was then undertaken in seven patients. Clinically, all were reported to be satisfied with the results, but one showed no correction after a mean follow-up of two years. The authors concluded that every case should be treated individually. In some feet an overcorrection at the time of surgery and additional soft-tissue releases may be helpful in preventing subsequent loss of correction.

In our study the rate of recurrence was nearly identical, four of 22 compared with one of seven, after a longer follow-up. In those feet in which correction was lost, this was not associated with a reduction in the length of the medial cuneiform or a lengthening of the cuboid, but with the development of increasing adduction of the forefoot, suggesting that the forefoot compensated for the effect of the osteotomy at the tarsal level.

Recurrence of adductus deformity has, so far, been observed after every form of treatment for club foot. ${ }^{10} \mathrm{We}$ observed the greatest loss of correction in patients with the longest follow-up. Although not statistically significant, this suggests a tendency to loss of correction with time after this procedure. We have not carried out overcorrection in these cases, as proposed by McHale and Lenhart. ${ }^{5}$ Neither our data nor those of by McHale and Lenhart ${ }^{5}$ allowed us to identify those patients at risk of recurrence of adductus at the time of surgery, and the long-term effect of overcorrection of a midfoot or forefoot deformity is unknown. In idiopathic club feet, operative overcorrection has been described as being the main disadvantage of complete subtalar release. ${ }^{11}$ Similarly, overcorrection of hallux valgus can result in hallux varus, which is difficult to manage. ${ }^{12}$ We therefore aim at a complete correction of the deformity by the double osteotomy and sometimes 
include a medial soft-tissue release. If the soft tissues are very rigid, we now apply an external fixator on the medial side of the foot before osteotomy to stretch the medial soft tissues.

In our study, all patients with idiopathic club feet were free from pain, satisfied with the operation and neither the surgeon nor the patients considered further correction to be necessary after a mean follow-up of five years. Recurrence of the deformity does not necessarily influence adversely the patient's satisfaction with the result. This suggests that the ability to wear normal shoes and the absence of pain are more important than the prevention of recurrence of the deformity.

Secondary club feet did not benefit from this operation. There was virtually no improvement of the adductus, although additional procedures and, in two feet, a second osteotomy were carried out. The soft-tissue abnormalities in these feet may be too severe to be corrected satisfactorily by this combined osteotomy. ${ }^{13}$ The Ilizarov technique has been recommended for difficult secondary club feet. Grill and Fanke ${ }^{14}$ advocated this method in 1987 for neglected club feet, but the only arthrogrypotic foot in their series had a complete relapse of the deformity. Brunner, Hefti and Tgetgel $^{15}$ treated 16 arthrogrypotic feet with a circular frame, of which 11 had a severe adductus deformity. In six patients, who had an osteotomy of the first metatarsal, correction was maintained, whereas in five without an osteotomy a significant loss of correction was observed. This suggests that the combination of an osteotomy with continuous soft-tissue distraction may be necessary to maintain correction in these feet. The Ilizarov technique seems to give the best results in these severe deformities, but the treatment is complex, involves fixation of the lower leg and usually lasts several months. ${ }^{16}$

Combined cuboid/cuneiform osteotomy is a safe operation, which allows satisfactory correction of residual adductus deformity in previously treated idiopathic club feet, but there is a potential for recurrence of the deformity. In secondary club feet, this technique alone is not appropriate.

No benefits in any form have been received or will be received from a commercial party related directly or indirectly to the subject of this article.

\section{References}

1. Kling TF, Conklin MJ, Schmidt TL. Surgical complications: adduction/supination. In: Simons GW, ed. The clubfoot: the present and $a$ view of the future. New York, Springer Verlag, 1993:404-12.

2. Tarraf YN, Carroll NC. Analysis of the components of residual deformity in clubfeet presenting for reoperation. J Pediatr Orthop 1992;12:207-16.

3. Kite JH. Congenital metatarsus varus. J Bone Joint Surg [Am] 1967;49-A:388-97.

4. Stark JG, Johanson JE, Winter RB. The Heyman-Herndon tarsometatarsal capsulotomy for metatarsus adductus: results in 48 feet. $J$ Pediatr Orthop 1987;7:305-10.

5. McHale KA, Lenhart MK. Treatment of residual clubfoot deformity - the 'bean-shaped' foot - by opening wedge medial cuneiform osteotomy and closing wedge cuboid osteotomy: clinical review and cadaver correlations. J Pediatr Orthop 1991;11:374-81.

6. Moulin P, Hefti F. Long-term results in the treatment of clubfoot. Orthopaede 1986;15:184-90.

7. Simons GW. Complete subtalar release in club feet. Part II: comparison with less extensive procedures. J Bone Joint Surg [Am] 1985;67-A:1056-65.

8. Meary R. On the measurement of the angle between the talus and the first metatarsal. Rev Chir Orthop 1967;53:390-419.

9. Downey D, Drennan J, Garcia J. Magnetic resonance imaging in congenital talipes equinovarus. In: Simons GW, ed. The clubfoot. The present and a view of the future. New York, Springer-Verlag, 1994:77-80

10. Vizkelety T, Szepesi K. Reoperation in treatment of clubfoot. $J$ Pediatr Orthop 1989;9:144-7.

11. Simons GW. Complete subtalar release in club feet. Part I. J Bone Joint Surg [Am] 1985;67-A:1044-55.

12. Richardson EG. The foot in adolescents and adults. In: Crenshaw $\mathrm{AH}$, ed. Campbell's operative orthopaedics. St Louis: CV Mosby, 1987:829-98.

13. Sodergard J, Ryoppy S. Foot deformities in arthrogryposis multiplex congenita. J Pediatr Orthop 1994;14:768-72.

14. Grill F, Fanke J. The Ilizarov distractor for the correction of relapsed or neglected clubfoot. J Bone Joint Surg [Br] 1987;69-B:593-7.

15. Brunner R, Hefti F, Tgetgel J. Arthrogrypotic joint contracture at the knee and the foot: correction with a circular frame. J Pediatr Orthop B 1997;6:192-7.

16. Grant AD, Atar D, Lehman WB. The Ilizarov technique in correction of complex foot deformities. Clin Orthop 1992;280:94-103. 